\title{
Decidability of the extension problem for maps into odd-dimensional spheres*
}

\author{
Lukáš Vokř́inek
}

June 26, 2018

\begin{abstract}
In a recent paper [3, it was shown that the problem of existence of a continuous map $X \rightarrow Y$ extending a given map $A \rightarrow Y$ defined on a subspace $A \subseteq X$ is undecidable, even for $Y$ an even-dimensional sphere. In the present paper, we prove that the same problem for $Y$ an odd-dimensional sphere is decidable. More generally, the same holds for any $d$-connected target space $Y$ whose homotopy groups $\pi_{k} Y$ are finite for $k>2 d$.
\end{abstract}

\section{Introduction}

The main object of study of the present paper is the extension problem. Given spaces $X, Y$ and a map $f: A \rightarrow Y$ defined on a subspace $A \subseteq X$, it questions the existence of a continuous extension

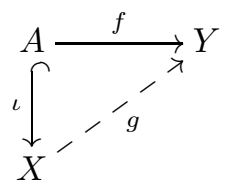

If $Y$ is allowed non-simply connected, this problem is undecidable by a simple reduction to the word problem in groups. Thus, we restrict ourselves to the situation of a simply connected $Y$.

In [8, Steenrod expressed a hope that the extendability problem would be algorithmically solvable. It was proved in [1] that this is indeed the case if one restricts to a suitably "stable" situation, i.e. if $\operatorname{dim} X \leq 2 \operatorname{conn} Y+1$. The algorithm of that paper depended on computations with abelian groups of homotopy classes of maps that are not available unstably. Later, the authors showed in 3 that the previous positive result was very much the best possible: the extension problem with $\operatorname{dim} X>2 \operatorname{conn} Y+1$ is undecidable, even for such a simple target space as $S^{d+1}$ with $d+1$ even. This undecidability result has implications to other problems, namely, [5] shows the undecidability of the problem of existence of a robust zero of a given PL-map $K \rightarrow \mathbb{R}^{d+2}$, again for $d$ even.

It may thus come as a bit of a surprise that the last two problems with $d+1$ odd are decidable - this is the content of Theorem 1 below. It applies to $Y=S^{d+1}, d+1$ odd, since in this case, $\pi_{n} S^{d+1}$ is finite for $n>d+1$. Again, [5] implies the decidability of the problem of existence of a robust zero of a given PL-map $K \rightarrow \mathbb{R}^{d+2}, d$ odd.

Theorem 1. There exists an algorithm that, given a pair of finite simplicial sets $(X, A)$, a finite $d$-connected simplicial set $Y, d \geq 1$, with homotopy groups $\pi_{n} Y$ finite for all $2 d<n<\operatorname{dim} X$ and a simplicial map $f: A \rightarrow Y$, decides the existence of a continuous extension $g: X \rightarrow Y$ of $f$.

*The research was supported by the grant P201/11/0528 of the Czech Science Foundation (GA ČR). 2010 Mathematics Subject Classification. Primary 55Q05; Secondary 55S35. Key words and phrases. Homotopy class, computation, higher difference. 
We do not have any bounds on the running time of such an algorithm. In the light of the \#P-hardness of the computation of the homotopy group $\pi_{k} Y$ when $k$ is a part of the input (in unary), see [3], one should not expect that this algorithm is polynomial-time when the dimension of $X$ is not fixed. However, even if $\operatorname{dim} X$ is bounded, it seems that our algorithm will not have polynomial running time. Nevertheless, the contrast with the undecidability for even-dimensional spheres is huge.

In Section 5, we briefly discuss an extension of Theorem 1 to the fibrewise equivariant situation of [4. In the special case $A=\emptyset$, such an extension implies the decidability of the problem of existence of a $\mathbb{Z} / 2$-equivariant map $X \rightarrow S^{d+1}$ when $d+1$ is odd. The index of $X$, denoted ind $X$, is the smallest $d+1$ for which such an equivariant map $X \rightarrow S^{d+1}$ exists; it has many applications in geometry and combinatorics. Thus, with the equivariant version of Theorem 11, it is possible to narrow ind $X$ down to two possible values.

\section{Sets with an action and mappings to abelian groups}

Let $S$ and $T$ be sets with a binary operation $+: S \times T \rightarrow S$ that has a right-sided zero $0 \in T$, i.e. such that $x+0=x$. We use the bracketing convention $x+y+z=(x+y)+z$. We define a "derived" action of $T$ on $S$ by

$$
x+\theta y=x+y+\cdots+y .
$$

Again, it has a right-sided zero 0. The following lemma will be our main technical tool.

Lemma 2. Let $f: S \rightarrow G$ be an arbitrary mapping of $S$ into an abelian group $G$. Then, for each prime power $q=p^{m}$ and $\ell_{0}>0$, there exists $\ell \geq \ell_{0}$, a function $D_{q, \ell} f: S \times T^{\ell} \rightarrow G$ such that $D_{q, \ell} f\left(x ; y_{1}, \ldots, y_{\ell}\right)=0$ whenever $y_{i}=0$ for some $i$, and $\theta>0$ such that

$$
f(x+\theta y) \equiv f(x)+D_{q, \ell} f(x ; y, \ldots, y) .
$$

In fact, $D_{q, \ell}$ is a formal expression in terms of $f$, the action of $T$ on $S$ and the group structure on $G$ and works universally for all $f: S \rightarrow G$. Moreover, this expresion is computable.

We will make a heavy use of higher-order differences

$$
\Delta_{\ell} f\left(x ; y_{1}, \ldots, y_{\ell}\right)=\sum_{\substack{0 \leq k \leq \ell \\ 1 \leq i_{1}<\cdots<i_{k} \leq \ell}}(-1)^{\ell-k} f\left(x+y_{i_{1}}+\cdots+y_{i_{k}}\right) .
$$

Clearly, $\Delta_{\ell} f\left(x ; y_{1}, \ldots, y_{\ell}\right)=0$ whenever $y_{i}=0$ for some $i$.

For any formal expression written in terms of the action of $T$ on $S$, we will use a superscript $(-)^{(\theta)}$ to denote the expression obtained by replacing each $x+y$ by $x+\theta y$. In this way, we yield $\Delta_{\ell}^{(\theta)} f$. The function $D_{q, \ell} f$ will be an integral combination of the $\Delta_{\ell}^{(\theta)} f$.

Proof. We let $\ell=p^{n}$ be any power of $p$ for which $\ell \geq \ell_{0}$ and $\theta=p^{n+m-1}$. The proof is executed by induction with respect to $m$. By definition, $f\left(x+p^{n+m-1} y\right)$ equals

$$
f\left(x+p^{n} p^{m-1} y\right)=\Delta_{\ell}^{\left(p^{m-1}\right)} f(x ; y, \ldots, y)-\sum_{j=0}^{p^{n}-1}(-1)^{p^{n}-j}\left(\begin{array}{c}
p^{n} \\
j
\end{array}\right) f\left(x+j p^{m-1} y\right) .
$$

For $j>0$, write $j=p^{n^{\prime}} j^{\prime}$ where $j^{\prime}$ is prime to $p$ and observe that

$$
j\left(\begin{array}{c}
p^{n} \\
j
\end{array}\right)=p^{n}\left(\begin{array}{c}
p^{n}-1 \\
j-1
\end{array}\right)
$$

is divisible by $p^{n}$, so that $p^{n-n^{\prime}} \mid\left(\begin{array}{c}p^{n} \\ j\end{array}\right)$. Setting $n^{\prime}+m=n+m^{\prime}$, we have either $m^{\prime} \leq 0$, in which case $n-n^{\prime} \geq m$ and the binomial coefficient is divisible by $q=p^{m}$, or we obtain for $q^{\prime}=p^{m^{\prime}}$ by induction

$$
f\left(x+j p^{m-1} y\right)=f\left(x+p^{n+m^{\prime}-1} j^{\prime} y\right) \equiv f(x)+D_{q^{\prime}, \ell}^{\left(j^{\prime}\right)} f(x ; y, \ldots, y) \quad\left(\bmod q^{\prime}\right)
$$


(this holds even for $j=0$ when the last term is interpreted as 0). Upon multiplication by $\left(\begin{array}{c}p^{n} \\ j\end{array}\right)$, that is divisible by $p^{n-n^{\prime}}=q / q^{\prime}$, we obtain even

$$
\left(\begin{array}{c}
p^{n} \\
j
\end{array}\right) f\left(x+j p^{m-1} y\right) \equiv\left(\begin{array}{c}
p^{n} \\
j
\end{array}\right) f(x)+\left(\begin{array}{c}
p^{n} \\
j
\end{array}\right) D_{q^{\prime}, \ell}^{\left(j^{\prime}\right)} f(x ; y, \ldots, y) .
$$

Since $\sum_{j=0}^{p^{n}-1}(-1)^{p^{n}-j}\left(\begin{array}{c}p^{n} \\ j\end{array}\right)=-1$, substituting the previous equation into the first yields

$$
\begin{aligned}
f\left(x+p^{n+m-1} y\right) & \equiv f(x)+\Delta_{\ell}^{\left(p^{m-1}\right)} f(x ; y, \ldots, y)-\sum_{j=0}^{p^{n}-1}(-1)^{p^{n}-j}\left(\begin{array}{c}
p^{n} \\
j
\end{array}\right) D_{q^{\prime}, \ell}^{\left(j^{\prime}\right)} f(x ; y, \ldots, y) \\
& =f(x)+D_{q, \ell} f(x ; y, \ldots, y),
\end{aligned}
$$

where we set $D_{q, \ell}=\Delta_{\ell}^{\left(p^{m-1}\right)}-\sum_{j=0}^{p^{n}-1}(-1)^{p^{n}-j}\left(\begin{array}{c}p^{n} \\ j\end{array}\right) D_{q^{\prime}, \ell}^{\left(j^{\prime}\right)}$.

Example 3. In this example, we have $q=p^{m}=4$ and $\ell=4$. Then

$$
f(x+8 y)=\Delta_{4}^{(2)} f(x ; y, y, y, y)+4 f(x+6 y)-6 f(x+4 y)+4 f(x+2 y)-f(x)
$$

and we continue in a similar way with the third term,

$$
f(x+4 y)=\Delta_{4}^{(1)} f(x ; y, y, y, y)+4 f(x+3 y)-6 f(x+2 y)+4 f(x+y)-f(x) .
$$

Substituting into the first equation, we get

$$
f(x+8 y) \equiv f(x)+\Delta_{4}^{(2)} f(x ; y, y, y, y)+2 \Delta_{4}^{(1)} f(x ; y, y, y, y)
$$

and $D_{4,4} f=\Delta_{4}^{(2)} f+2 \Delta_{4}^{(1)} f$.

\section{Postnikov tower}

We assume that $Y$ is $d$-connected simplicial set and has all homotopy groups $\pi_{n} Y$ finite for $2 d<n<\operatorname{dim} X=D$. In the following theorem, $K(\pi, n+1)$ is the Eilenberg-MacLane space and $E(\pi, n)$ its path space; more precisely, we use the canonical minimal models with both simplicial sets minimal and the projection $\delta: E(\pi, n) \rightarrow K(\pi, n+1)$ a minimal fibration, see [7].

Theorem 4. For each simply connected simplicial set $Y$, it is possible to construct simplicial sets $P_{n}$, for $n<D$, and a sequence of simplicial maps

$$
Y \stackrel{\varphi_{n}}{\longrightarrow} P_{n}
$$

such that $\varphi_{n *}: \pi_{i}(Y) \rightarrow \pi_{i}\left(P_{n}\right)$ is an isomorphism for $i \leq n$ and $\pi_{i}\left(P_{n}\right)=0$ for $i>n$.

Further, for $2 d<n<D$, it is possible to construct simplicial sets $P_{n, i}$ that fit into a pullback square

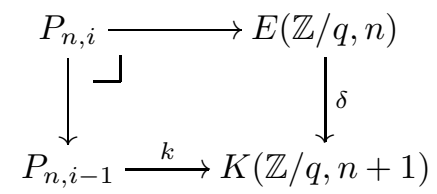

with $q=p^{m}$ a prime power (depending on $n$ and $i$; the same applies to $k$ ) and $P_{n-1}=P_{n, 0}, P_{n}=$ $P_{n, r}$, where $r$ is some integer that depends on $n$. The composition of the projections $P_{n, i} \rightarrow P_{n, i-1}$ for $i=1, \ldots, r$ is a map $p_{n}: P_{n} \rightarrow P_{n-1}$ for which $p_{n} \varphi_{n}=\varphi_{n-1}$. 
Proof. The paper [2] gives the simplicial sets $P_{n}$. To obtain their refinements $P_{n, i}$, we compute a decomposition

$$
\pi_{n} \cong \mathbb{Z} / q_{1} \oplus \cdots \oplus \mathbb{Z} / q_{r}
$$

of the $n$-th homotopy group into a sum of cyclic groups of prime power orders. Then we define $\pi_{n, i}=\mathbb{Z} / q_{1} \oplus \cdots \oplus \mathbb{Z} / q_{i}$ with obvious projections pr: $\pi_{n} \rightarrow \pi_{n, i} ; P_{n, i}$ is the following pullback

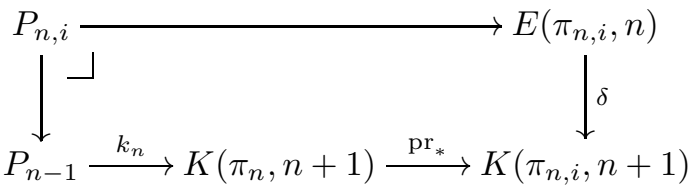

Theorem 5. It is possible to construct an action $x+\Theta y, \Theta \gg 0$, of $P_{2 d}$ on each $P_{n, i}$, for $2 d \leq n<D$, that has a right-sided zero $0 \in P_{2 d}$. The projections $P_{n, i} \rightarrow P_{n, i-1}$ respect this action.

Proof. We will construct, by induction with respect to $n$ and $i$, positive integers $\Theta_{n, i}$ and an action $x+\Theta_{n, i} y$ of $P_{2 d}$ on $P_{n, i}$. The action $x+\Theta y$ from the statement is then obtained by setting $\Theta=\Theta_{D-1, r}$ and deriving the action $\Theta_{n, i}$; this is possible since $\Theta_{n, i} \mid \Theta$ by construction. Starting with $n=2 d$, the paper [1] constructs an abelian H-group structure on $P_{2 d}$, i.e. an action of $P_{2 d}$ on itself; we set $\Theta_{2 d+1,0}=1$.

For the induction step, we apply Lemma2 to the Postnikov invariant $k: P_{n, i-1} \rightarrow K(\mathbb{Z} / q, n+1)$ - its target is a simplicial abelian group, i.e. an abelian group in each dimension. The function

$$
D_{q, \ell}^{\left(\Theta_{n, i-1}\right)} k: P_{n, i-1} \times P_{2 d} \times \cdots \times P_{2 d} \rightarrow K(\mathbb{Z} / q, n+1)
$$

(formally, it is not derived from $D_{q, \ell} k$ since $x+y$ is not defined, but we want to emphasize that it is with respect to the action $\left.x+\Theta_{n, i-1} y\right)$ is zero whenever at least one of the components in $P_{2 d}$ is zero and thus we have a diagram

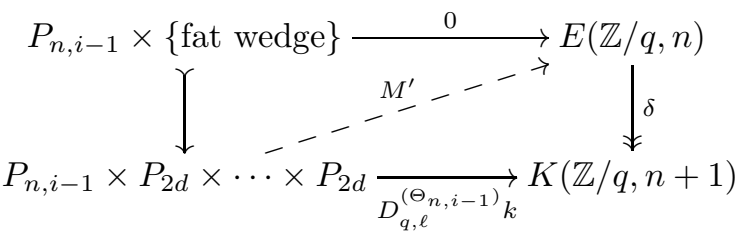

(the fat wedge consists of those $\ell$-tuples $\left(y_{1}, \ldots, y_{\ell}\right) \in P_{2 d} \times \cdots \times P_{2 d}$ with at least one $y_{i}$ equal to the basepoint 0$)$. The cofibre of the map on the left is $\left(P_{n, i-1}\right)_{+} \wedge P_{2 d} \wedge \cdots \wedge P_{2 d}$ and is $(\ell(d+1)-1)$-connected. Therefore, when $\ell \gg 0$, a diagonal $M^{\prime}$ exists; it can be computed as in [1]. We define $M(x, y)=M^{\prime}(x ; y, \ldots, y)$, so that

$$
\delta M(x, y)=D_{q, \ell}^{\left(\Theta_{n, i-1}\right)} k(x ; y, \ldots, y)=k\left(x+\theta \Theta_{n, i-1} y\right)-k(x),
$$

where $\theta$ is the output of Lemma 2. Denoting $\Theta_{n, i}=\theta \Theta_{n, i-1}$, this allows us to define a new action on $P_{n, i} \subseteq P_{n, i-1} \times E(\mathbb{Z} / q, n)$ by the formula

$$
(x, c)+\Theta_{n, i} y=\left(x+\Theta_{n, i} y, c+M(x, y)\right)
$$

(the compatibility holds since $\delta(c+M(x, y))=\delta c+\delta M(x, y)=k(x)+\left(k\left(x+\Theta_{n, i} y\right)-k(x)\right)=$ $\left.k\left(x+\Theta_{n, i} y\right)\right)$.

After the following simple observation, we will be ready to prove Theorem 1

Lemma 6. For each $g^{\prime}: X \rightarrow P_{2 d}$ and $2 d<n<D$, it is possible to compute the finite set of homotopy classes of all lifts $g: X \rightarrow P_{n}$.

Proof. This follows from the fact that each $\pi_{n}$ is finite for $2 d<n<D$. Namely, since $\pi_{2 d+1}$ is finite, the number of all lifts of $g^{\prime}$ to a map $X \rightarrow P_{2 d+1}$ is finite. Thus, it is possible to go through all these partial lifts and compute all their lifts to $P_{n}$ by recursion. 


\section{Proof of Theorem 1}

For $n=D-1$, let $f: A \rightarrow P_{n}$ also denote the composition $A \stackrel{f}{\rightarrow} Y \stackrel{\varphi_{n}}{\rightarrow} P_{n}$. By the usual obstruction theory, it is enough to check whether an extension to $g: X \rightarrow P_{n}$ exists - the higher obstructions are all zero. Thus, we consider the Postnikov stage $P_{n}$ with an action $x+\Theta y$ by the stage $P_{2 d}$. Consider the commutative square (the $R$ and $R^{\prime}$ are the restriction maps while $\Pi_{X}$ and $\Pi_{A}$ are post-compositions with the projection $P_{n} \rightarrow P_{2 d}$ )

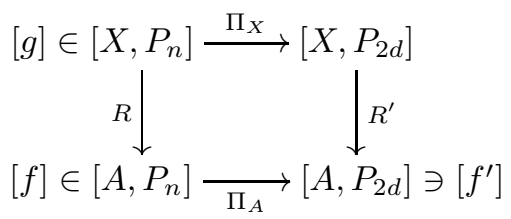

with $\left[f^{\prime}\right]=\Pi_{A}[f]$. We compute the groups on the right explicitly as in [1] and consider the subset $H=\left(R^{\prime}\right)^{-1}\left[f^{\prime}\right]$ of all possible extensions of $f^{\prime}$ to a map $X \rightarrow P_{2 d}$. There is a finite set $H_{0} \subseteq H$ such that $H=H_{0}+\Theta$ ker $R^{\prime}$; namely, if $\left[h_{0}\right] \in H$ and we identify $\operatorname{ker} R^{\prime} \cong \mathbb{Z} / q_{1} \oplus \cdots \oplus \mathbb{Z} / q_{r}$ (possibly with some $q_{i}=0$ giving $\mathbb{Z} / 0=\mathbb{Z}$ ), we may take for $H_{0}$ all $r$-tuples of the form $\left[h_{0}\right]+\left(z_{1}, \ldots, z_{r}\right) \in H$ with each $\left|z_{i}\right| \leq \Theta / 2$.

Suppose first that $g$ is any extension of $f$ and express its image in $\left[X, P_{2 d}\right]$ as $\Pi_{X}[g]=[h]-\Theta[k]$ with $[h] \in H_{0}$ and $[k] \in \operatorname{ker} R^{\prime}$. Then $[\widehat{g}]=[g]+\Theta[k] \in \Pi_{X}^{-1}\left(H_{0}\right)$ also gives an extension of $f$ since

$$
R[\widehat{g}]=R([g]+\Theta[k])=[f]+\Theta R^{\prime}[k]=[f],
$$

(the operations in homotopy classes are natural and $[k] \in \operatorname{ker} R^{\prime}$ ). Thus, we see that an extension $g$ exists if and only if $[f] \in R \Pi_{X}^{-1}\left(H_{0}\right)$. This set is finite and its representatives can be computed using Lemma 6. For each $[\widehat{f}] \in R \Pi_{X}^{-1}\left(H_{0}\right)$, we may then test whether $[\widehat{f}]=[f]$ by the main theorem of [6].

\section{A fibrewise equivariant version}

The same argument could be repeated in the fibrewise equivariant setup of [4, though actions with a strict right-sided zero have to be replaced by ones with a weak zero. Denoting $I=\Delta^{1}$, this structure is a map

$$
\left(1 \times P_{n, i} \times_{B} P_{2 d}\right) \cup\left(I \times P_{n, i} \times_{B} B\right) \rightarrow P_{n, i}
$$

consisting of an action and a homotopy $x \sim x+0$.

The most significant difference lies in the proof of Theorem 5 . The space $P_{n, i-1} \times P_{2 d} \times \cdots \times P_{2 d}$ has to be replaced by the following subspace of $I^{\ell} \times\left(P_{n, i-1} \times{ }_{B} P_{2 d} \times{ }_{B} \cdots \times{ }_{B} P_{2 d}\right)$ :

$$
\bigcup_{\substack{k \geq 0, 1 \leq i_{1}<\cdots<i_{k} \leq \ell}}\left(d_{i_{1}}^{+} \cdots d_{i_{k}}^{+} I^{\ell}\right) \times\left(P_{n, i-1} \times{ }_{B} \vee_{B}^{i_{1}, \ldots, i_{k}} P_{2 d}\right),
$$

where $d_{i_{1}}^{+} \cdots d_{i_{k}}^{+} I^{\ell} \subseteq I^{\ell}$ consists of those $\ell$-tuples $\left(t_{1}, \ldots, t_{\ell}\right)$ with $t_{i_{1}}=\cdots=t_{i_{k}}=1$ and where $\vee_{B}^{i_{1}, \ldots, i_{k}} P_{2 d} \subseteq P_{2 d} \times{ }_{B} \cdots \times_{B} P_{2 d}$ is formed by those $\ell$-tuples $\left(y_{1}, \ldots, y_{\ell}\right)$ whose components $y_{j}$ with $j \notin\left\{i_{1}, \ldots, i_{k}\right\}$ lie on the zero section $B$. In particular, $\vee_{B}^{\emptyset} P_{2 d}=B \times_{B} \cdots \times_{B} B$ and $\vee_{B}^{1, \ldots, \ell} P_{2 d}=P_{2 d} \times_{B} \cdots \times_{B} P_{2 d}$.

The subspace $P_{n, i-1} \times\{$ fat wedge $\}$ is replaced by the subspace of (11) formed by those elements whose component in $I^{\ell}$ has at least one component equal to 0 . By the methods of [4, it is then easy to equip this pair with effective homology, compute the variation of the map $M^{\prime}$ from the proof of Theorem 5 and use it to define a new weak action of $P_{2 d}$ on $P_{n, i}$. 


\section{References}

[1] M. Čadek, M. Krčál, J. Matoušek, F. Sergeraert, L. Vokř́nek, U. Wagner. Computing all maps into a sphere. Preprint, arXiv:1105.6257, 2011. Extended abstract in Proc. ACM-SIAM Symposium on Discrete Algorithms (SODA 2012), 1-10.

[2] M. Čadek, M. Krčál, J. Matoušek, L. Vokř́nek, U. Wagner. Polynomial-time computation of homotopy groups and Postnikov systems in fixed dimension. Preprint, arXiv:1211.3093, 2012.

[3] M. Čadek, M. Krčál, J. Matoušek, L. Vokřínek, U. Wagner. Extendability of continuous maps is undecidable. Preprint, arXiv:1302.2370, 2013.

[4] M. Čadek, M. Krčál, L. Vokř́nek. Algorithmic solvability of the lifting-extension problem. Preprint, arXiv:1307.6444, 2013.

[5] P. Franek, M. Krčál. Robust Satisfiability of Systems of Equations. Preprint, http://kam.mff.cuni.cz/ krcal/pmwiki/uploads/Main/soda.pdf 2013. To appear in Proc. ACM-SIAM Symposium on Discrete Algorithms (SODA 2014).

[6] M. Filakovský, L. Vokřínek. Are two given maps homotopic? An algorithmic viewpoint. Preprint, arXiv:1312.2337, 2013.

[7] J. P. May. Simplicial Objects in Algebraic Topology. University of Chicago Press, Chicago, IL, 1992.

[8] N. E. Steenrod. Cohomology operations and obstructions to extending continuous functions: colloquium lectures. Princeton University, 1957.

LUKÁŠ VOKŘÍNEK

Department of Mathematics and Statistics,

Masaryk University,

Kotlářská 2, 61137 Brno,

Czech Republic

koren@math.muni.cz 